\title{
On computing the complex passivity radius
}

\author{
Michael L. Overton and Paul Van Dooren
}

\begin{abstract}
We characterize the complex passivity radius of a rational transfer matrix $G(s):=C\left(s I_{n}-A\right)^{-1} B+D$ and propose an approach to compute it. The method depends on computing the smallest structured indefinite perturbation to a Hermitian matrix that makes it singular. We consider both additive and multiplicative perturbations, giving details for the additive case. In both cases, the smallest indefinite perturbation can be efficiently computed by solving a unimodal optimization problem in a real parameter. The passivity radius can be computed by minimizing the smallest singularity-inducing multiplicative indefinite perturbation of a frequency-dependent matrix over the imaginary axis.
\end{abstract}

\section{INTRODUCTION}

We consider a time-invariant continuous-time system with an $m \times m$ transfer matrix given by a minimal realization $G(s):=C\left(s I_{n}-A\right)^{-1} B+D$ where $A \in \mathbb{C}^{n \times n}, B \in \mathbb{C}^{n \times m}$, $C \in \mathbb{C}^{m \times n}$, and $D \in \mathbb{C}^{m \times m}$. Such a transfer function is said to be passive if it is stable and if the Hermitian part of $G(j \omega)$ is positive definite on the imaginary axis, i.e. if :

$$
\Re \lambda_{i}(A)<0, \quad G(j \omega)+[G(j \omega)]^{*} \succ 0,
$$

for all eigenvalues $\lambda_{i}(A)$ and for all frequencies $\omega$. If we now consider the perturbed transfer function $G_{\Delta}(s):=C_{\Delta}\left(s I_{n}-\right.$ $\left.A_{\Delta}\right)^{-1} B_{\Delta}+D_{\Delta}$ where

$$
\left[\begin{array}{ll}
A_{\Delta} & B_{\Delta} \\
C_{\Delta} & D_{\Delta}
\end{array}\right]:=\left[\begin{array}{cc}
A & B \\
C & D
\end{array}\right]-\left[\begin{array}{cc}
\Delta_{A} & \Delta_{B} \\
\Delta_{C} & \Delta_{D}
\end{array}\right]
$$

we can ask the question: when is passivity is lost as the norm of the perturbation increases? More precisely we consider complex perturbations of the form

$$
\Delta:=\left[\begin{array}{cc}
\Delta_{A} & \Delta_{B} \\
\Delta_{C} & \Delta_{D}
\end{array}\right]
$$

and wish to compute

$$
\inf \left\{\|\Delta\|_{2} \mid G_{\Delta}(s) \text { is not passive }\right\} .
$$

We call this quantity the passivity radius of the system $G(s)$.

Since the eigenvalues of $A_{\Delta}$ and $G_{\Delta}(j \omega)+\left[G_{\Delta}(j \omega)\right]^{*}$ vary continuously with $\Delta$, it follows that the passivity of $G_{\Delta}(s)$ is lost when either $\Re \lambda_{i}\left(A_{\Delta}\right)=0$ or

$$
\operatorname{det}\left(G_{\Delta}(j \omega)+\left[G_{\Delta}(j \omega)\right]^{*}\right)=0 \text { for some } \omega \in \mathbb{R} .
$$

One can show that when passivity is lost then (1) must certainly hold [2] (and loss of stability might happen simultaneously). Moreover, the zeros of the determinant (1) can

M. L. Overton is with the Courant Institute of Mathematical Sciences New York University, NY 10012, U.S.A.(overton@ cs . nyu . edu)

P. Van Dooren is with CESAME, Université catholique de Louvain, B1348 Louvain-la-Neuve, Belgium (vdooren@csam.ucl.ac.be) be obtained from

$$
\operatorname{det}\left(\left[\begin{array}{ccc}
0 & B_{\Delta} & A_{\Delta}-j \omega I_{n} \\
B_{\Delta}^{*} & D_{\Delta}+D_{\Delta}^{*} & C_{\Delta} \\
A_{\Delta}^{*}+j \omega I_{n} & C_{\Delta}^{*} & 0
\end{array}\right]\right)=0
$$

since the matrix in (1) is the Schur complement of the matrix above. Equation (2) is a Hamiltonian generalized eigenvalue problem and its imaginary eigenvalues can be computed efficiently [1].

In order to extract the perturbation matrix $\Delta$, we rewrite this determinant condition as

$$
\operatorname{det}\left(\mathscr{H}(\omega)-E\left[\begin{array}{c|c}
0 & \Delta \\
\hline \Delta^{*} & 0
\end{array}\right] E^{T}\right)=0,
$$

where $\mathscr{H}(\omega)$ is a Hermitian matrix for all $\omega$ :

$$
\mathscr{H}(\omega):=\left[\begin{array}{ccc}
0 & B & A-j \omega I_{n} \\
B^{*} & D+D^{*} & C \\
A^{*}+j \omega I_{n} & C^{*} & 0
\end{array}\right],
$$

and where

$$
E:=\left[\begin{array}{cccc}
I_{n} & 0 & 0 & 0 \\
0 & I_{m} & 0 & I_{m} \\
0 & 0 & I_{n} & 0
\end{array}\right] .
$$

This is also equivalent to the determinant condition

$$
\operatorname{det}\left(I_{2 p}-\left[\begin{array}{c|c}
0 & \Delta \\
\hline \Delta^{*} & 0
\end{array}\right] H(\omega)\right)=0,
$$

where

$$
p=m+n, \quad H(\omega):=E^{T} \mathscr{H}(\omega)^{-1} E .
$$

For a fixed value of $\omega$, finding the perturbation $\Delta$ of smallest 2-norm that makes this matrix singular is a matrix perturbation problem that we address in the next section.

\section{STRUCTURED INDEFINITE PERTURBATIONS}

In this section we look at structured perturbations of a given Hermitian matrix. We first look at structured additive perturbations $\Delta_{H}$ of a given matrix $H$ that make $H-\Delta_{H}$ singular. Specifically, we partition $H$ and $\Delta H$ as follows:

$$
H=\left[\begin{array}{cc}
S & R \\
R^{*} & T
\end{array}\right], \quad \Delta_{H}=\left[\begin{array}{cc}
0 & \Delta_{R} \\
\Delta_{R}^{*} & 0
\end{array}\right],
$$

where $S$ and $T$ are $n \times n$ Hermitian matrices. We then consider structured multiplicative perturbations $\Delta_{H}$ of the same form that make $I-\Delta_{H} H$ singular. 


\section{A. Existence of solution}

We first consider additive perturbations $\Delta_{H}$. Let $H-\Delta_{H}$ be singular. Then this perturbed matrix has a non-zero null vector $z$, which we partition accordingly :

$$
\left(H-\Delta_{H}\right) z=\left[\begin{array}{cc}
S & R-\Delta_{R} \\
R^{*}-\Delta_{R}^{*} & T
\end{array}\right]\left[\begin{array}{l}
u \\
v
\end{array}\right]=0 .
$$

Multiplying this from the left by $\left[u^{*},-v^{*}\right]$ and then taking the real part, we obtain the necessary condition $u^{*} S u=$ $v^{*} T v$ for the existence of a singularity-inducing Hermitian perturbation of the type (5). If $S$ is positive definite and $T$ is negative definite (or vice versa), then there is obviously no solution. In fact, the converse also holds, as the following theorem states.

Theorem 1 Let $H$ be a nonsingular Hermitian matrix. Then there exists an additive perturbation $\Delta H$ of the type (5) such that $\operatorname{det}\left(H-\Delta_{H}\right)=0$ if and only if the matrix

$$
\left[\begin{array}{cc}
S & 0 \\
0 & -T
\end{array}\right]
$$

is not (positive or negative) definite.

Proof: The above discussion shows that if there exists a non-zero vector $z$ in the kernel of $H-\Delta_{H}$ then it follows that $u^{*} S u=v^{*} T v$, or equivalently

$$
z^{*}\left[\begin{array}{cc}
S & 0 \\
0 & -T
\end{array}\right] z=0,
$$

which implies that (6) is a necessary condition. To show sufficiency we transform the matrix $H-\Delta_{H}$ as follows :

$$
\begin{gathered}
\hat{H}-\Delta_{\hat{H}}:=\left[\begin{array}{cc}
D_{S} & \hat{R}-\Delta_{\hat{R}} \\
\hat{R}^{*}-\Delta_{\hat{R}}^{*} & D_{T}
\end{array}\right] \\
=\left[\begin{array}{cc}
U_{S}^{*} & 0 \\
0 & U_{T}^{*}
\end{array}\right]\left[\begin{array}{cc}
S & R-\Delta_{R} \\
R^{*}-\Delta_{R}^{*} & T
\end{array}\right]\left[\begin{array}{cc}
U_{S} & 0 \\
0 & U_{T}
\end{array}\right]
\end{gathered}
$$

where $U_{S}$ and $U_{T}$ are unitary, and where $D_{S}$ and $D_{T}$ are the diagonal matrices of eigenvalues of $S$ and $T$, respectively. The condition (6) implies that there must exist a pair of diagonal elements $s_{i}$ and $t_{k}$ with non-negative product (i.e. zero or positive). Then choose $\Delta_{\hat{R}}$ such that the $i$-th row and $k$-th column of $\hat{R}-\Delta_{\hat{R}}$ are zero except for the $(i, k)$ element, which we choose as $\sqrt{s_{i} t_{k}}$. This clearly makes $\hat{H}-$ $\Delta_{\hat{H}}$ singular, and therefore also $H-\Delta_{H}$.

The analogous result for multiplicative perturbations is slightly more complicated. We state a sufficient condition without proof.

Theorem 2 Let $H$ be a nonsingular Hermitian matrix. A sufficient condition for there to exist a multiplicative perturbation $\Delta H$ of the type (5) such that $\operatorname{det}\left(I-\Delta_{H} H\right)=0$ is that there exist vectors $u$ and $v$ such that $u^{*} S u=v^{*} T v \neq 0$.

\section{B. Minimum norm perturbation}

We will use in this section a perturbation result of Weyl that we first recall here (see [3] for a proof). Throughout the remainder of the paper, denote the ordered eigenvalues of any $m \times m$ Hermitian matrix $M$ by $\lambda_{1}(M) \geq \cdots \geq \lambda_{m}(M)$.
Lemma 3 (Weyl) Let $M$ and $\hat{M}$ be two $n \times n$ Hermitian matrices. Then

$$
\|M-\hat{M}\|_{2} \geq\left|\lambda_{i}(M)-\lambda_{i}(\hat{M})\right|,
$$

for $i=1, \ldots, m$.

For any Hermitian matrix $M$, define

$$
\begin{aligned}
\underline{\mu}(M) & :=\min _{i}\left\{\lambda_{i}(M): \lambda_{i}(M)>0\right\}, \\
\underline{\nu}(M) & :=\min _{i}\left\{-\lambda_{i}(M): \lambda_{i}(M)<0\right\}
\end{aligned}
$$

where we adopt the convention that minimization over the empty set yields $\infty$. A first bound for the minimal perturbation $\Delta_{H}$ that makes $\operatorname{det}\left(H-\Delta_{H}\right)=0$ is easily stated. Since the $\left|\lambda_{i}(H)\right|$ are the singular values of $H$, the following result is immediate, but we point out that it also follows from Weyl's result (which we shall need later).

Lemma 4 Let $H$ be a nonsingular Hermitian matrix. Then the smallest Hermitian perturbation $\Delta H$ such that $H-\Delta H$ is singular is given by

$$
\min \left\|\Delta_{H}\right\|_{2}=\min \{\underline{\mu}(H), \underline{v}(H)\}
$$

and a perturbation achieving this is given by $\Delta_{H}=\lambda_{i}(H) z z^{*}$, where $\lambda_{i}(H)$ is an eigenvalue corresponding to the minimum in (8) and $z$ is a corresponding normalized eigenvector.

Proof: The bound is a consequence of Weyl's theorem since $\hat{H}:=H-\Delta_{H}$ has a zero eigenvalue.

If we now impose a constraint of the type (5) on the perturbation then it is unlikely that $\Delta_{H}$ given by Lemma 4 will satisfy the constraint and hence we can expect the minimal structured perturbation $\Delta_{H}$ to have a larger norm than (8). Following [6], we consider the scaled matrix

$$
H_{\gamma}:=\left[\begin{array}{cc}
\gamma^{2} S & R \\
R^{*} & T / \gamma^{2}
\end{array}\right]=\Gamma\left[\begin{array}{cc}
S & R \\
R^{*} & T
\end{array}\right] \Gamma
$$

where

$$
\Gamma:=\left[\begin{array}{cc}
\gamma I_{n} & 0 \\
0 & I_{n} / \gamma
\end{array}\right]
$$

and $\gamma$ is real and positive. Observe that this transformation leaves the perturbation $\Delta_{H}$ invariant, so

$$
\Gamma\left(H-\Delta_{H}\right) \Gamma=H_{\gamma}-\Delta_{H} .
$$

Thus for all $\gamma \in(0, \infty)$, the inertia of $H_{\gamma}-\Delta_{H}$ equals the inertia of $H-\Delta_{H}$, and in particular whether or not $H_{\gamma}-\Delta_{H}$ is singular is independent of $\gamma$. Hence $\Delta_{H}$ must satisfy a bound similar to that of Lemma 4 for all values of $\gamma$. We make this more precise in the following two theorems.

Theorem 5 Let $H$ be a nonsingular Hermitian matrix. Then the smallest perturbation $\Delta H$ with the structure given in (5) such that $H-\Delta_{H}$ is singular is bounded by

$$
\inf \left\|\Delta_{H}\right\|_{2} \geq \sup _{\gamma} \min \left\{\underline{\mu}\left(H_{\gamma}\right), \underline{v}\left(H_{\gamma}\right)\right\} .
$$

Proof: The proof follows immediately by applying Lemma 3 to $H_{\gamma}$ for all values of $\gamma$. Since the inequality (8) holds for all $\gamma$ in the open interval $0<\gamma<\infty$, it must also hold for the supremum. 
Fig. 1. Plot of the singular values of $H_{\gamma}$ as a function of $\gamma$

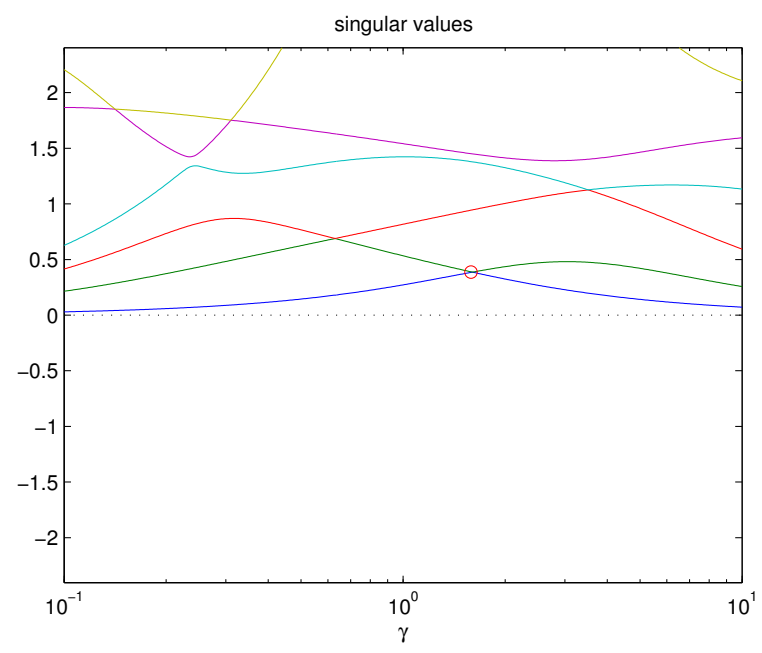

Fig. 1 illustrates Theorem 5. The singular values $\left|\lambda_{i}\left(H_{\gamma}\right)\right|$ (not the eigenvalues) are plotted as a function of $\gamma$ from 0.1 to 10 for a randomly generated Hermitian matrix $H$. The circle in the graph shows the lower bound given by Theorem 5, namely the maximum least singular value over $\gamma$, achieved at, say, $\gamma=\hat{\gamma}$. However, the theorem does not exhibit a perturbation that has the required structure. In fact, this is not generally possible when the smallest singular value coincides locally with one eigenvalue $\mu\left(H_{\gamma}\right)$ on one side of $\hat{\gamma}$ and a different eigenvalue $-\underline{v}\left(H_{\gamma}\right)$ on the other side of $\hat{\gamma}$, as is the case in Fig. 1. This is demonstrated by the following stronger result, illustrated in Fig. 2, where the eigenvalues $\lambda_{i}\left(H_{\gamma}\right)$ are plotted over the same range for $\gamma$ and for the same matrix $H$. The circle in Fig. 2 illustrates the lower bound given by Theorem 6, namely the smaller of the absolute values of the maximum value of the least positive eigenvalue $\mu\left(H_{\gamma}\right)$ and the minimum of the largest negative eigenvalue $-\bar{v}\left(H_{\gamma}\right)$. Notice that, since $H$ is Hermitian, the situation illustrated in Fig. 2, namely that the eigenvalues are smooth functions of $\gamma$, is generic, since the codimension of the manifold of complex Hermitian matrices (resp. real symmetric matrices) with multiple eigenvalues is 3 (resp. 2) and $H_{\gamma}$ is a oneparameter family (see [5], where a figure like Fig. 2 appears on the cover of the text). On the other hand, Fig. 1 is also generic, since the only requirement is that the generically smooth curves $\mu$ and $\underline{v}$ cross at some value of $\gamma$. Note that the proof of Theorem 6 includes the nongeneric case where eigenvalues coincide for some $\gamma$ : there is no assumption that the functions $\lambda_{i}\left(H_{\gamma}\right), \mu\left(H_{\gamma}\right)$ and $\underline{v}\left(H_{\gamma}\right)$ are smooth functions of $\gamma$.

Theorem 6 Let $H$ be a nonsingular Hermitian matrix. Then the smallest perturbation $\Delta H$ with the structure given in (5) such that $\operatorname{det}\left(H-\Delta_{H}\right)=0$ is bounded by

$$
\inf \left\|\Delta_{H}\right\|_{2} \geq \min \left\{\sup _{\gamma} \underline{\mu}\left(H_{\gamma}\right), \sup _{\gamma} \underline{v}\left(H_{\gamma}\right) \cdot\right\}
$$

Proof: Consider a graph of the eigenvalues $\lambda_{i}\left(H_{\gamma}\right)$ as
Fig. 2. Plot of the eigenvalues of $H_{\gamma}$ as a function of $\gamma$

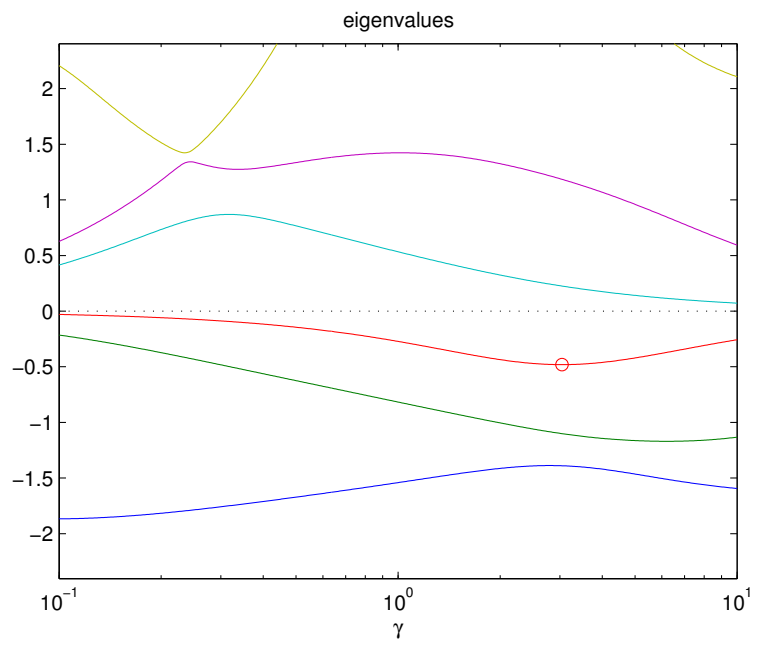

a function of $\gamma$ as in Fig. 2. These curves are continuous but not necessarily smooth. Since the matrix $H_{\gamma}$ is nonsingular for all $\gamma \in(0, \infty)$ these eigenvalue curves do not intersect the horizontal axis. Furthermore, if $H_{\gamma}-\Delta_{H}$ is singular for some $\gamma$, it must be singular for all $\gamma \in(0, \infty)$, and since the inertia of $H_{\gamma}-\Delta_{H}$ is independent of $\gamma$, it follows that there is at least one index $i$ for which the eigenvalue $\lambda_{i}\left(H_{\gamma}-\Delta_{H}\right)$ is identically zero for all $\gamma$ (not shown in figure). Thus, by Weyl's theorem,

$$
\left\|\Delta_{H}\right\|_{2} \geq\left|\lambda_{i}\left(H_{\gamma}\right)-\lambda_{i}\left(H_{\gamma}-\Delta_{H}\right)\right|=\left|\lambda_{i}\left(H_{\gamma}\right)-0\right| .
$$

Suppose that $\lambda_{i}\left(H_{\gamma}\right)$ is positive (recall that the sign is the same for all $\gamma$ ). Then it follows that

$$
\left|\lambda_{i}\left(H_{\gamma}\right)\right| \geq \underline{\mu}\left(H_{\gamma}\right)
$$

and since this is independent of $\gamma$ we obtain

$$
\left\|\Delta_{H}\right\|_{2} \geq \sup _{\gamma} \underline{\mu}\left(H_{\gamma}\right) .
$$

Similarly, if $\lambda_{i}\left(H_{\gamma}\right)$ is negative we have

$$
\left|\lambda_{i}\left(H_{\gamma}\right)\right| \geq \underline{v}\left(H_{\gamma}\right)
$$

and therefore

$$
\left\|\Delta_{H}\right\|_{2} \geq \sup _{\gamma} \underline{v}\left(H_{\gamma}\right) .
$$

Since one bound or the other must hold, we have the desired result.

It follows from Theorem 6 that if both $\sup _{\gamma} \underline{\mu}\left(H_{\gamma}\right)$ and $\sup _{\gamma} \underline{v}\left(H_{\gamma}\right)$ are unbounded, there is no solution to the problem. Indeed, if e.g. $S \succ 0$ and $T \prec 0$ then the sub-matrix $\gamma^{2} S$ of $H_{\gamma}$ guarantees that it has $n$ positive eigenvalues tending to $+\infty$ for $\gamma$ going to $+\infty$ and the sub-matrix $T / \gamma^{2}$ guarantees that it has $n$ negative eigenvalues tending to $-\infty$ as $\gamma$ goes to 0 . Thus both suprema are unbounded.

The next theorem shows that the bound just proved is tight. The proof exhibits a $\Delta_{H}$ that solves the problem, but we do not give all the details. 
Theorem 7 Let $H$ be a nonsingular Hermitian matrix. Then the smallest perturbation $\Delta H$ with the structure given in (5) such that $\operatorname{det}\left(H-\Delta_{H}\right)=0$ has norm given by

$$
\min \left\|\Delta_{H}\right\|_{2}=\min \left\{\sup _{\gamma} \underline{\mu}\left(H_{\gamma}\right), \sup _{\gamma} \underline{v}\left(H_{\gamma}\right)\right\}
$$

when the right hand side is bounded.

Proof: Somewhat analogously to the proof in [6], we observe that there are three possible cases when the righthand side is bounded; we give details only for the first case. The first case occurs when the relevant supremum is achieved by an eigenvalue $\lambda_{i}\left(H_{\hat{\gamma}}\right)$ for some index $i$ and some $\hat{\gamma} \in(0, \infty)$ and this eigenvalue is simple (does not coincide with any other $\left.\lambda_{k}\left(H_{\hat{\gamma}}\right)\right)$. It follows that $\lambda_{i}\left(H_{\gamma}\right)$ is differentiable with respect to $\gamma$ at $\hat{\gamma}$, with

$$
\frac{d \lambda_{i}\left(H_{\gamma}\right)}{d \gamma}=z^{*} \frac{d H_{\gamma}}{d \gamma} z=0,
$$

where $z$ is a corresponding normalized eigenvector [3]. Observe that

$$
\frac{d H_{\gamma}}{d \gamma}=2 \gamma^{-1}\left(D H_{\gamma}+H_{\gamma} D\right)
$$

where $D=\operatorname{diag}\left\{I_{n},-I_{n}\right\}$. If we partition $z=\left[u^{*} v^{*}\right]^{*}$, then at $\gamma=\hat{\gamma}$,

$z^{*} \frac{d H_{\gamma}}{d \gamma} z=2 \lambda_{i}\left(H_{\gamma}\right) \gamma^{-1} z^{*} D z=2 \lambda_{i}\left(H_{\gamma}\right) \gamma^{-1}\left(\|u\|_{2}^{2}-\|v\|_{2}^{2}\right)=0$.

Thus $\|u\|_{2}^{2}=\|v\|_{2}^{2}=1 / 2$. We now construct $\Delta_{H}$ by setting $\Delta_{R}=2 \lambda_{i}\left(H_{\hat{\gamma}}\right) u v^{*}$, for which $\left\|\Delta_{H}\right\|_{2}=\left|\lambda_{i}\left(H_{\hat{\gamma}}\right)\right|$ and $H_{\hat{\gamma}}-\Delta_{H}$ has the null vector $z$. The equation (12) follows from this and the lower bound established by Theorem 6 .

The second case occurs when the lower supremum in (12) is achieved by an eigenvalue $\lambda_{i}\left(H_{\hat{\gamma}}\right)$ for some index $i$ and some $0<\hat{\gamma}<\infty$ and this eigenvalue coincides with one or more $\lambda_{k}\left(H_{\hat{\gamma}}\right), k \neq i$. In this case one shows that there exists an eigenvector $z=\left[\begin{array}{ll}u^{*} & v^{*}\end{array}\right]^{*}$ in the corresponding invariant subspace such that $\|u\|_{2}=\|v\|_{2}$, but we omit the details.

The third case is that the lower supremum in (12) converges to a limiting value as $\gamma \rightarrow 0$ or $\gamma \rightarrow \infty$. This case can occur only when $S$ and $T$ are both positive semidefinite (or both negative semidefinite), and at least one of them is singular. In this case the minimizing perturbation can be constructed directly; we omit the details.

We now observe that the distance characterized by Theorem 7 can be computed efficiently since either $\mu$ or $\underline{v}$, whichever has a lower supremum, is unimodal. The beautifully simple proof is again inspired by [6]. Note that we do not claim that $\mu$ and $\underline{v}$ are both unimodal functions. In fact, this is not always the case.

Theorem 8 Let $H$ be a nonsingular Hermitian matrix and suppose that at least one of the suprema in the right-hand side of (12) is finite. If the minimum of the two suprema is the supremum of $\underline{\mu}\left(H_{\gamma}\right)$, then any local extremum of $\underline{\mu}\left(H_{\gamma}\right)$ is a global maximum. Likewise, if the minimum of the two suprema is the supremum of $\underline{v}\left(H_{\gamma}\right)$, then any local extremum of $\underline{v}\left(H_{\gamma}\right)$ is a global maximum.
Proof: Without loss of generality, suppose the first of the two cases holds. Suppose that $\tilde{\gamma}$ locally minimizes or maximizes $\mu\left(H_{\gamma}\right)$. The proof of theorem 7 applies in exactly the same way to $\tilde{\gamma}$ and exhibits a perturbation $H_{\Delta}$ for which $\left\|\Delta_{H}\right\|_{2}=\left|\lambda_{i}\left(H_{\tilde{\gamma}}\right)\right|$ and $H_{\tilde{\gamma}}-\Delta_{H}$ is singular. If there were another $\gamma$ for which $\mu\left(H_{\gamma}\right)$ is larger, this would violate Theorem 6.

The multiplicative perturbation results are similar. For any Hermitian matrix $M$, define

$$
\begin{aligned}
\bar{\mu}(M) & :=\max _{i}\left\{\lambda_{i}(M): \lambda_{i}(M)>0\right\}, \\
\bar{v}(M) & :=\max _{i}\left\{-\lambda_{i}(M): \lambda_{i}(M)<0\right\},
\end{aligned}
$$

with the convention that maximizing over the empty set yields $-\infty$. The main result for multiplicative perturbations is as follows.

Theorem 9 Let $H$ be a Hermitian matrix. Then the smallest perturbation $\Delta H$ with the structure given in (5) such that $\operatorname{det}\left(I-\Delta_{H} H\right)=0$ has norm given by

$$
\min \left\|\Delta_{H}\right\|_{2}=1 / \max \left\{\inf _{\gamma} \bar{\mu}\left(H_{\gamma}\right), \inf _{\gamma} \bar{v}\left(H_{\gamma}\right)\right\},
$$

when the denominator on the right-hand side is nonzero.

A proof of this theorem for invertible $H$ is immediately obtained by applying Theorem 7 to $H^{-1}$. We omit the proof for singular $H$ here.

\section{COMPUTING THE PASSIVITY RADIUS}

As a consequence of an analogy of Theorem 8 for the multiplicative case, the solution characterized by Theorem 9 can be computed efficiently. Each univariate minimization problem can be solved using bisection since derivatives are available. One minimization may fail because the function is not unimodal, but if this occurs, it must be the other infimum that is larger. Determining which computed minimizer is correct is straightforward.

It follows from the previous section that in order to compute the passivity radius $r_{P}$, one needs to compute

$$
r_{P}^{-1}=\max _{\omega} \max \left\{\operatorname { i n f } _ { \gamma } \overline { \mu } \left(H_{\gamma}(\omega), \inf _{\gamma} \bar{v}\left(H_{\gamma}(\omega)\right\}\right.\right.
$$

where

$$
H(\omega):=\Gamma E^{T} \mathscr{H}(\omega)^{-1} E \Gamma .
$$

We point out here that the right-hand side of (15) is nonzero for all $\omega$ because the sufficient condition of Theorem 2 is always satisfied. The $(1,1)$ and $(2,2)$ blocks of $H(\omega):=$ $E^{T} \mathscr{H}(\omega)^{-1} E$ are given by

$$
\begin{aligned}
& H_{1,1}=X(\omega)^{*}\left(G(j \omega)+[G(j \omega)]^{*}\right)^{-1} X(\omega), \\
& H_{2,2}=Y(\omega)^{*}\left(G(j \omega)+[G(j \omega)]^{*}\right)^{-1} Y(\omega),
\end{aligned}
$$

where

$$
\begin{gathered}
X(\omega):=\left[-C\left(A-j \omega I_{n}\right)^{-1}, I_{m}\right] \\
Y(\omega):=\left[I_{m},-B^{T}\left(A^{T}+j \omega I_{n}\right)^{-1}\right] .
\end{gathered}
$$

These matrices correspond to the matrices $S$ and $T$ of Theorem 2 and clearly have the same inertia. 
The expression (15) is a two-parameter optimization problem. We propose to solve this using a level set method. This is inspired by a method given in [7] to compute the real stability radius, solving the two-parameter optimization problem described in [6].

In order to explain the principle, we assume for simplicity that

$$
r_{P}^{-1}=\max _{\omega} \tau^{*}(\omega)
$$

where

$$
\tau^{*}(\omega):=\inf _{\gamma} \tau_{\gamma}(\omega), \quad \tau_{\gamma}(\omega)=\bar{\mu}\left(H_{\gamma}(\omega)\right) .
$$

We start by choosing a frequency $\omega_{o}$ and compute $\tau^{*}\left(\omega_{o}\right)$. Let $\gamma_{o}$ be a minimizing value of $\gamma$; it can be shown that $\gamma_{0} \in(0, \infty)$. Now freeze $\gamma_{0}$ and observe that

$$
\xi_{o}:=\tau_{\gamma_{o}}\left(\omega_{o}\right)=\tau^{*}\left(\omega_{o}\right)
$$

but at all other frequencies $\omega$ we have

$$
\tau_{\gamma_{o}}(\omega) \geq \tau^{*}(\omega)
$$

One then computes the intervals of $\omega$ for which $\tau_{\gamma_{o}}(\omega)>\xi_{o}$ via the solution of an eigenvalue problem (see [7]). These are obtained from the real zeros $\omega$ of

$$
\operatorname{det}\left(\xi_{o} I_{2 p}-E_{\gamma_{o}}^{T} \mathscr{H}(\omega)^{-1} E_{\gamma_{o}}\right)=0,
$$

where

$$
E_{\gamma_{o}}:=\left[\begin{array}{cccc}
\gamma_{o} I_{n} & 0 & 0 & 0 \\
0 & \gamma_{o} I_{m} & 0 & I_{m} / \gamma_{o} \\
0 & 0 & I_{n} / \gamma_{o} & 0
\end{array}\right]
$$

These are also the real zeros $\omega$ of the equation

$$
\operatorname{det}\left(\mathscr{H}(\omega)-E_{\gamma_{o}} \xi_{o}^{-1} E_{\gamma_{o}}^{T}\right)=0,
$$

which is a Hamiltonian generalized eigenvalue problem :

$$
\left[\begin{array}{ccc}
-\gamma_{o}^{2} I_{n} / \xi_{o} & B & A-j \omega I_{n} \\
B^{*} & D+D^{*}-\left(\gamma_{o}^{2}+\gamma_{o}^{-2}\right) I_{m} / \xi_{o} & C \\
A^{*}+j \omega I_{n} & C^{*} & -\gamma_{o}^{-2} I_{n} / \xi_{o}
\end{array}\right] .
$$

These so-called level sets are the only intervals in which we can find $\max _{\omega} \tau^{*}(\omega)$ because of (16). The algorithm proceeds to find a new frequency $\omega_{1}$ for which $\xi_{1}:=\tau^{*}\left(\omega_{1}\right)>\xi_{0}$ unless the union of the intervals is empty. The repeated application of this idea is shown in [7] to yield a sequence of levels $\xi_{i}$ that globally converges to the solution of the two-parameter optimization problem. Moreover, variants are described in [7], [4] for which the asymptotic convergence behavior was reported to be at least quadratic.

\section{THE REAL CASE}

We conclude by pointing out that if $\{A, B, C, D\}$ are real and the perturbation matrix $\Delta$ is also restricted to be real, the problem is considerably more involved : the matrix $H(\omega)$ is still complex and one has to consider real symmetric perturbations of a complex Hermitian matrix. This problem is not solved in this paper, and seems quite challenging. We illustrate this by analyzing the very simple $2 \times 2$ case. Let

$$
H:=\left[\begin{array}{cc}
s & r \\
r & t
\end{array}\right], \quad \Delta_{H}:=\left[\begin{array}{ll}
0 & \delta \\
\delta & 0
\end{array}\right]
$$

be a complex Hermitian matrix, and a real indefinite perturbation matrix, respectively. The smallest real perturbation $\delta$ that causes $\operatorname{det}\left(H-\Delta_{H}\right)$ to become zero must clearly satisfy

$$
s t=(r-\delta)(\bar{r}-\delta),
$$

which is a quadratic equation in $\delta$. Notice that $s$ and $t$ are real since $H$ is Hermitian. Denoting $r=x+j y$, the equation above becomes

$$
s t-y^{2}=(x-\delta)^{2},
$$

which has a solution iff $s t-y^{2} \geq 0$. For the case of complex perturbations $\delta$ the same $2 \times 2$ problem would have had a solution iff $s t \geq 0$ which is clearly less restrictive. The case of real indefinite perturbations of a complex Hermitian matrix is currently under investigation.

\section{ACKNOWLEDGEMENTS}

The authors would like to thank Radu Stefan for contributions to section I, and Yurii Nesterov and Frédéric Crevecoeur for several useful discussions. Michael Overton was supported in part by CORE, Université catholique de Louvain and in part by the U.S. National Science Foundation under grant DMS-0412049. Paul Van Dooren was supported by the Belgian Programme on Interuniversity Attraction Poles, initiated by the Belgian Federal Science Policy Office, and in part by the U.S. National Science Foundation under the ITR grant ACI-03-24944. The scientific responsibility rests with the authors.

\section{REFERENCES}

[1] P. Benner, V. Mehrmann and H. XU, A numerically stable, structure preserving method for computing the eigenvalues of real Hamiltonian or symplectic pencils, Numerische Mathematik, vol. 78(3), pp. 329-358, 1998.

[2] Y. Genin, Y. Hachez, Y. Nesterov, R. Stefan, P. Van Dooren, S. XU, Positivity and linear matrix inequalities, European Journal of Control, Vol. 8(3), pp. 275-298, 2002.

[3] R. Bhatia, Perturbation Bounds for Matrix Eigenvalues, Longman, Essex and Wiley, New York, 1987.

[4] C. Lawrence, A. Tits, P. Van Dooren, A fast algorithm for the computation of an upper bound on the $\mu$-norm, Automatica, vol. 36, pp. 449-456, 2000.

[5] P.D. LAX, Linear Algebra, Wiley, 1996.

[6] L. QiU, B. Bernhardsson, A. RANTzer, E.J. DAVison, P.M. Young, And J.C. DoYle, A formula for computation of the real stability radius, Automatica, vol. 31, pp. 879-890, 1995.

[7] J. Sreedhar, P. VAn Dooren, A.L. Tits, A fast algorithm to compute the real structured stability radius, in Int. Series of Numer. Math., Birkhauser, vol. 121, pp. 219-230, 1996. 